\title{
White light from an electroluminescent diode made from poly[3(4-octylphenyl)-2,2'- bithiophene] and an oxadiazole derivative
}

\author{
Magnus Berggren, Göran Gustafsson, Olle Inganäs, Mats R. Andersson, \\ T. Hjertberg and O. Wennerström
}

\section{Linköping University Post Print}

N.B.: When citing this work, cite the original article.

Original Publication:

Magnus Berggren, Göran Gustafsson, Olle Inganäs, Mats R. Andersson, T. Hjertberg and O. Wennerström, White light from an electroluminescent diode made from poly[3(4octylphenyl)-2,2'-bithiophene] and an oxadiazole derivative, 1994, Journal of Applied Physics, (76), 11, 7530-7534.

http://dx.doi.org/10.1063/1.357984

Copyright: American Institute of Physics (AIP) http://www.aip.org/

Postprint available at: Linköping University Electronic Press

http://urn.kb.se/resolve?urn=urn:nbn:se:liu:diva-74935 


\title{
White light from an electroluminescent diode made from poly[3(4-octylphenyl)-2,2' -bithiophene] and an oxadiazole derivative
}

\author{
$M$. Berggren, G. Gustafsson, and O. Inganäs \\ IFM, University of Linköping, S-581 83 Linköping, Sweden. \\ M. R. Andersson, a),b) T. Hjertberg, ${ }^{\text {b) }}$ and O. Wennerström ${ }^{\text {a) }}$ \\ Chalmers University of Technology, S-412 96 Göteborg, Sweden.
}

(Received 25 April 1994; accepted for publication 22 August 1994)

\begin{abstract}
We report on an electroluminescent diode emitting red, green, and blue light simultaneously. The device is based on a thin polymer layer, poly[3-(4-octylphenyl)-2,2 $2^{\prime}$-bithiophene] and a thick molecular layer, 2-(4-biphenylyl)-5-(4-tertbutyl-phenyl)1,3,5-oxadiazole. The quantum efficiency for light conversion is $0.3 \%$ and the turn-on voltage for light emission is $7 \mathrm{~V}$. In this article we present electric and spectroscopic characterizations. A mechanism for the light emission, based on electron and hole recombination between the two organic layers, is proposed. (C) 1994 American Institute of Physics.
\end{abstract}

\section{INTRODUCTION}

Light-emitting diodes (LEDs) made from organic materials have generated a lot of interest lately. ${ }^{1-5}$ The possibilities of making large area LEDs and of tuning the colors throughout the visible spectrum are especially attractive properties since it would make a full color display possible. Both polymers and molecules have been used in the construction of devices. The most general structure of an organic LED is that of an emitting layer sandwiched between two electrodes, one of which is electron injecting and the other hole injecting. In some devices so called hole transport and electron transport layer have been utilized to create single or double heterostructure devices. It has been shown that this significantly enhances the quantum efficiency of the device. ${ }^{5-9}$

In this work we have utilized a luminescent electron transport material, 2-(4-biphenylyl)-5(4-tertbutyl-phenyl) 1,3,4-oxadiazole (PBD) together with a conjugated luminescent polymer, poly[3-(4-octylphenyl)-2,2'-bithiophene] (PTOPT), to construct a heterojunction device. Similar devices have been made before with other conjugated polymers in combination with significantly thinner PBD layers. ${ }^{8,9}$ In these devices the quantum efficiency was increased by a factor of 5-10 compared to devices without a PBD layer. All these devices were single layer emitters. However, in our device we see emission from both layers as well as a peak which cannot be assigned to any fundamental transitions in the pure materials. Multiple peak emission has also been demonstrated in mixtures between different dyes in a polymer matrix. ${ }^{10,11}$

In this article we report on the results of the characterization of these devices as well as a discussion on the different mechanisms for light generation.

\section{EXPERIMENT}

The structure of the device is shown in Fig. 1. It consists of two active organic layers sandwiched between an electron injecting (aluminum) and a hole injecting contact (indium-tin oxide, ITO). The first layer is a soluble poly(3-(4-octylphenyl)-2,2'-bithiophene) (PTOPT) (Fig. 2) which was spin-coated onto an ITO coated glass slide (Balzer, Baltracon ${ }^{\mathrm{TM}}, 10 \Omega / \mathrm{sq}$ ) from a $2 \mathrm{mg} / \mathrm{ml}$ chloroform solution at $700-3000 \mathrm{rpm}$. This gives films of $400-800 \AA$ thicknesses. The synthesis of PTOPT is reported in Ref. 12. The second layer consists of 2-(4-biphenylyl)-5-(4-tertbutylphenyl)1,3,4-oxadiazole (PBD) (Fig. 2) (Aldrich Co. Ltd., used as supplied) which was deposited by evaporation $\left(P<10^{-6}\right.$ Torr). The thickness of the PBD layer was in the range of $1100-1700 \AA$. As the last step in the device fabrication a $400 \AA$ aluminum layer was evaporated $\left(P<10^{-6}\right.$ Torr) onto the molecular layer through a shadow mask. The active area of the devices were $2 \times 3 \mathrm{~mm}^{2}$. All sample preparation and handling was carried out in laboratory atmosphere.

Electrical and radiometric characterization of the device was made with a Keithley 617 programmable electrometer and a Hamamatsu silicon photo diode (1010BR) together with a Keithley 485 picoamperemeter respectively. External quantum efficiency, emitted photons per injected electrons, was measured using this photo diode. The area of the photo diode is $100 \mathrm{~mm}^{2}$ and it is placed $7 \mathrm{~mm}$ above the LED. We assume isotropic emission of the LED and the response of the photodiode has also been taken into account. Luminescence spectra were taken with an Oriel Instaspec 1B diode array spectrometer. All spectra were corrected for the re-

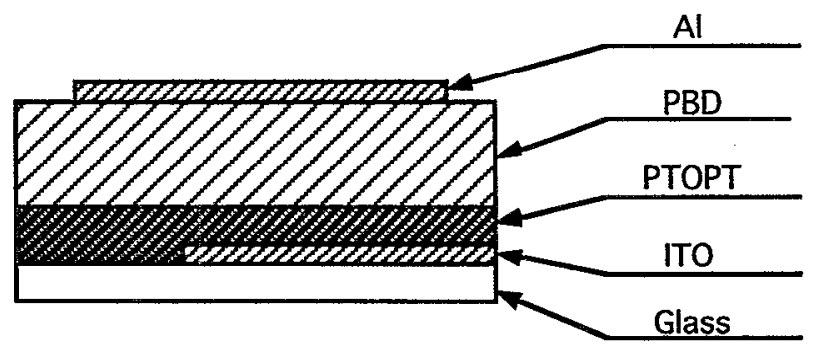

a) Department of Organic Chemistry.

${ }^{b)}$ Department of Polymer Technology.

FIG. 1. The diode structure. 

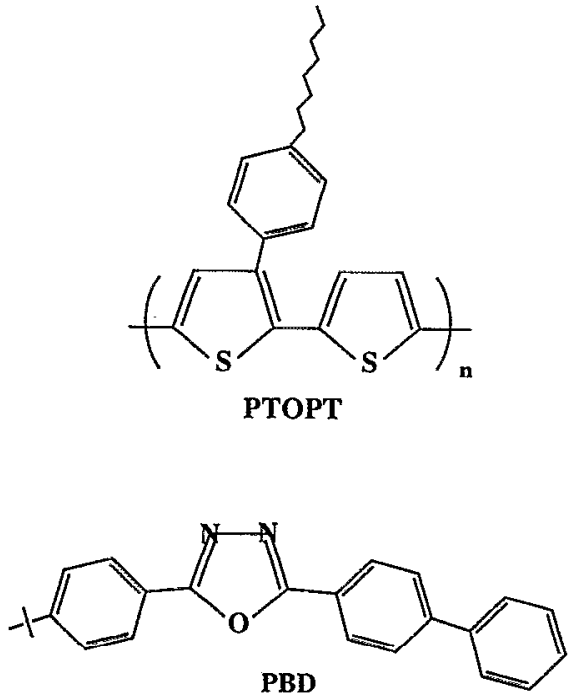

FIG. 2. The polymer (PTOPT) poly[3-(4-octylphenyl)-2,2'-bithiophene] and 2-(4-biphenylyl)-5-(4-tertbutyl-phenyl)1,3,4-oxadiazole (PBD).

sponse of the spectrometer system. The film thicknesses was measured with a Sloan-Dektak 3030 profilometer.

\section{RESULTS AND DISCUSSION}

Current Voltage $(I-V)$ characteristics of an ITO/ PTOPT $(500 \AA) / \mathrm{PBD}(1100 \AA) / \mathrm{Al}$ diode are given in Fig. 3 . The rectifying ratio at $\pm 12 \mathrm{~V}$ is 100 and the nonmonotonic behavior for negative voltage is probably due to short currents. The electroluminescence (EL) spectrum of an ITO/ $\operatorname{PTOPT}(500 \AA) / \mathrm{PBD}(1700 \AA) / \mathrm{Al}$ device under $18 \mathrm{~V}$ forward bias is shown in Fig. 4. The spectrum is characterized by three peaks, one at $410 \mathrm{~nm}$ (blue), one at 530 (green) and one at $610 \mathrm{~nm}$ (red-orange). To the eye, the color of this diode is bluish white. In the figure are also shown the photoluminescence (PL) spectra of PTOPT and PBD. As can be seen the EL peak at $410 \mathrm{~nm}$ resembles the PL emission from PBD. We therefore attribute this part of the EL spectrum to emission from the PBD layer. Similarly, the peak at $610 \mathrm{~nm}$ can

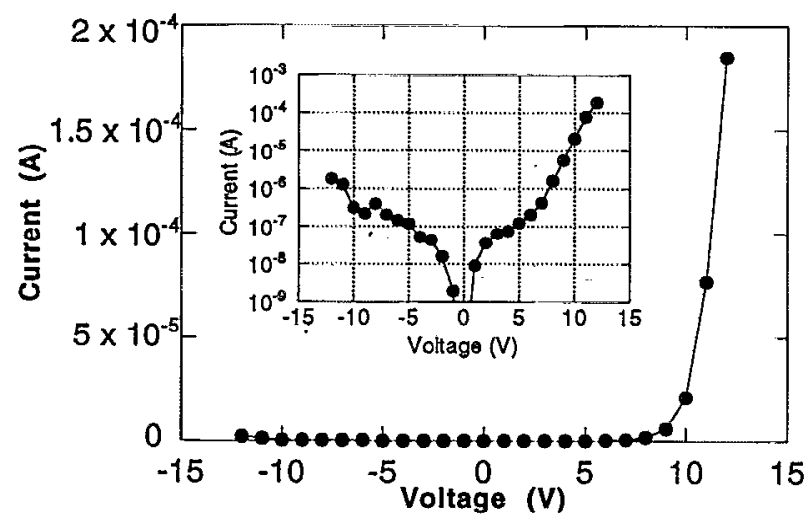

FIG. 3. $I-V$ characteristics of an ITO/PTOPT( $400 \AA) / \mathrm{PBD}(1100 \AA) / \mathrm{Al}$ device.

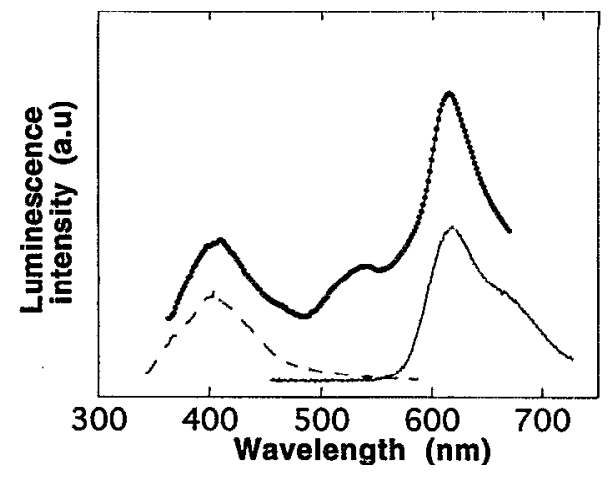

FIG. 4. Electroluminescence spectrum of an ITO/PTOPT/PBD/Al device (filled circles) under forward bias $(18 \mathrm{~V})$. In the figure is also shown the photoluminescence spectrum of PBD (dashed) and PTOPT (solid), respectively.

be attributed to radiative decay of singlet excitons in the PTOPT layer. The ITO/PBD/PTOPT/Al device is thus an example of a device emitting from a molecular layer and a polymer layer simultaneously.

The peak at $530 \mathrm{~nm}$ cannot be explained by singlet recombination in PBD or PTOPT. The peak is probably due to a transition at the interface between PBD and PTOPT. There are several possible origins to the peak; a transition from exciplexes formed at the interface, transitions frum band to impurity levels present at the interface, or a radiative transfer of electrons from PBD to hole-polarons in PTOPT. The peak is not present in PL spectra of ITO/PTOPT/PBD structures, glass/PBD structures, or in blends of PBD/PTOPT. We have not observed a shift of the green peak for different voltages. The difference between the photo- and the electroluminescence experiments is that in the former case the electron-hole pairs are present both in the bulk of the two materials and at the interface while in the latter they are probably concentrated at the interface region. However, in the blend the ratio of interface to bulk material is increased and transitions from impurities or from exciplexes should appear in the spectra if they were the dominating recombinations. Electron-hole pairs created by carrier injection can conceivably be created in either of the layers. The enhanced concentrations of either species at the interface between the hole transport and the electron transport layer, in combination with the high electric field, may allow transitions to occur that are only infrequent under photoluminescence conditions. Radiative transfer of electrons from PBD to hole-polarons in PTOPT is therefore more likely in electroluminescence than in photoluminescence.

Transitions originating from electron and holes in adjacent layers in semiconductor superlattices is known both from photoluminescence ${ }^{13}$ and electroluminescence. ${ }^{14}$ Our major difference to such structures is of course that both the polymer and molecule layer are quite different from the semiconductor crystal layers found in superlattices. Reports of exciplex photoluminescence from a polymer layer/ molecule layer assembly recently appeared in the literature. ${ }^{15}$ In this case a new PL peak intermediate to that of the two layer materials appeared, and the photoluminescence inten- 

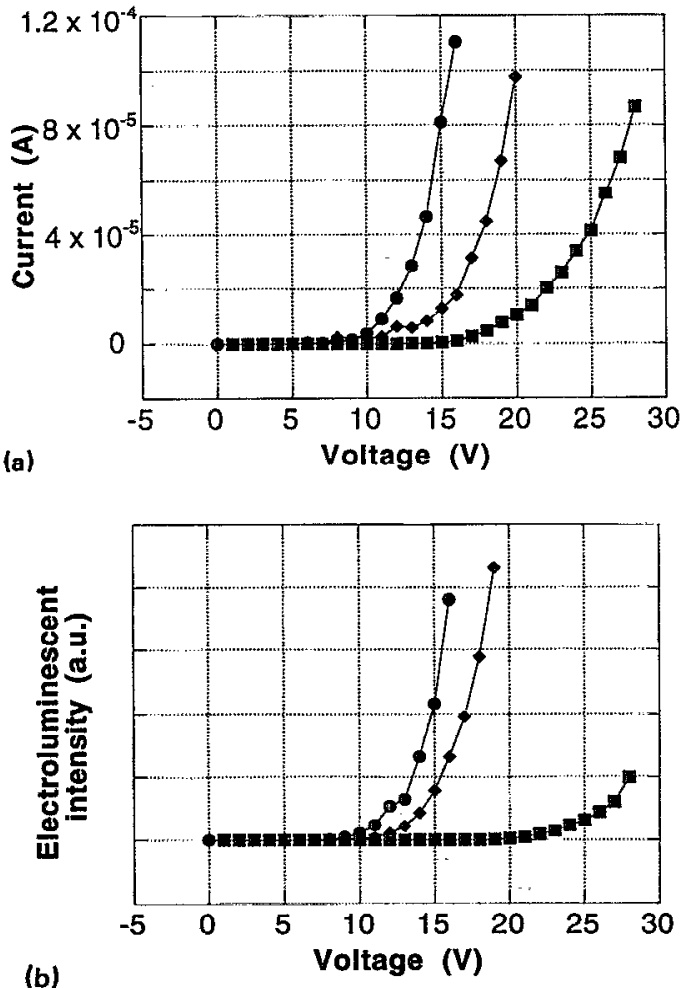

FIG. 5. Current vs voltage (a) and electroluminescence vs voltage (b) characteristics of an ITO/PTOPT/PBD/Al device with varying PBD thickness and $400 \AA$ PTOPT. The PBD thicknesses are $1100 \AA$ (circles), $1300 \AA$ (diamonds), and $1100 \AA$ (squares), respectively.

sity was greatly enhanced. The difference in our results is that we do not observe the green peak under photoluminescence conditions.

Figure 5 shows the current versus voltage $(I-V)$ characteristics and the electroluminescence versus voltage (EL- $V$ ) characteristics of ITO/PTOPT/PBD/Al devices with different PBD thicknesses. Positive bias is when a positive voltage is applied to the ITO electrode. As can be seen the current and the electroluminescent intensity are dependent on the PBD thickness. Figure 6 shows the $I-V$ and EL- $V$ characteristics of the same type of devices but with different PTOPT thicknesses. In this case there is a no pronounced thickness dependence. This indicates that the electric field in all these devices is mainly over the PBD film. These results support the model proposed by Brown et al. ${ }^{9}$ where positive charge is easily injected into the polymer film and then transported to the PBD/polymer interface, where they build up a space charge layer. This space charge increases the electric field over the PBD film and decreases the field over the polymer layer.

This mechanism would also imply an increased quantum efficiency (QE), since there is an improved balance between the injection of holes and electrons, respectively. This has already been observed in several structures where thin PBD layers were used. ${ }^{8,9}$ Figure 6 shows that this is also the case in our devices where we use considerably thicker PBD layers $(1300 \AA)$. For an ITO/PTOPT $(400 \AA) / \mathrm{Al}$ devicc a $\mathrm{QE}$ of $0.003 \%$ has been measured. The QE increases up to $0.3 \%$ when using the PBD layer. Figure 7 also shows that the
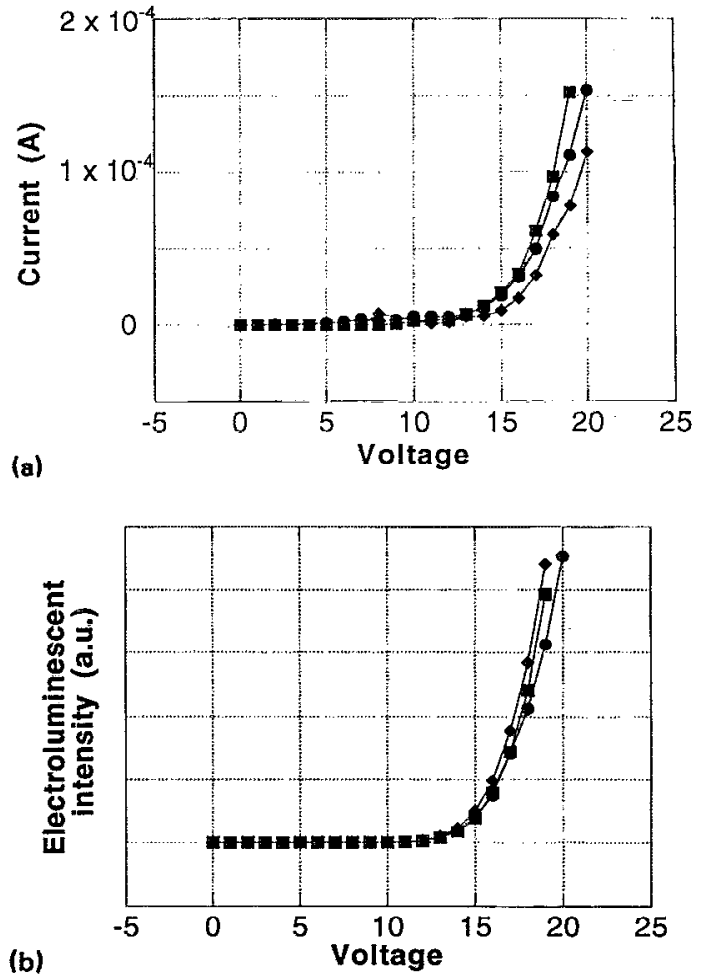

FIG. 6. Current vs voltage (a) and electroluminescence vs voltage (b) characteristics of an ITO/PTOPT/PBD/Al device with varying PTOPT thickness and $1300 \AA$ PBD layer. The PTOPT thicknesses are $200 \AA$ (circles), $340 \AA$ (diamonds), and $780 \AA$ (squares), respectively.

PTOPT layer is important for the efficiency of electroluminescence in PBD. Without the PTOPT layer only a negligible light intensity is detected from PBD. This suggests that PTOPT is acting as a hole transport layer in the device. This is further supported by the observation that the current density in a ITO/PTOPT/PBD/Al device is almost an order of magnitude higher than in a ITO/PBD/Al device, at equivalent voltages.

Similar results have been obtained in a multilayer structure, ITO/ $\alpha$-sexithienyl/sexiphenyl/Tris(8-hydroxyquinolino) aluminum(Alq/Mg:Ag) structure where the thiophene oligo-

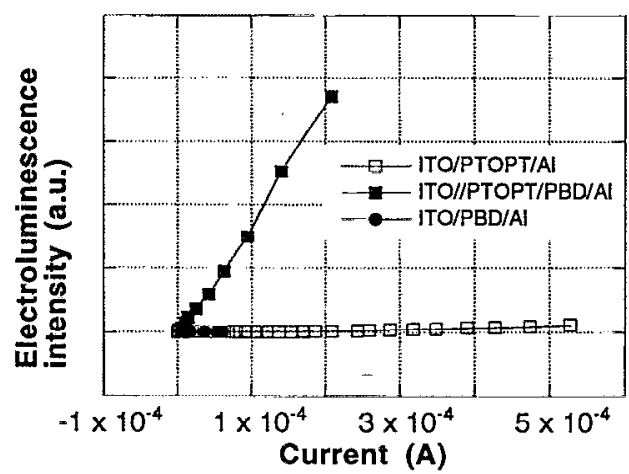

FIG. 7. Electroluminescence vs current of an ITO/PTOPT/PBD/Al device (filled squares), an ITO/PTOPT/Al device (open squares) and an ITO/ $\mathrm{PBD} / \mathrm{Al}$ device (tilled circles), respectively. 


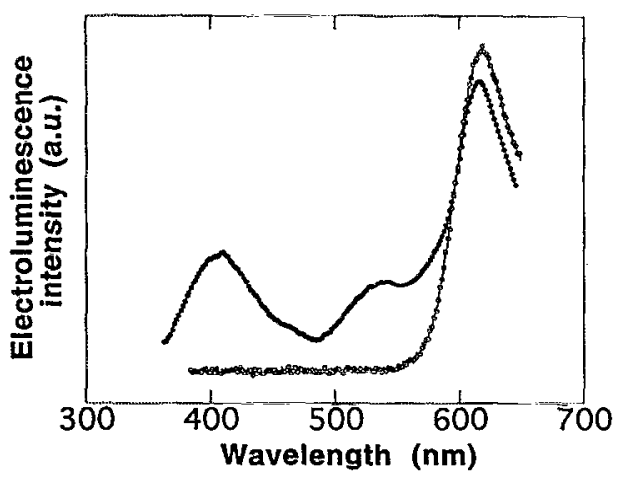

FIG. 8. Electroluminescence spectra of an ITO/PTOPT/PBD/Al device immediately after applying $18 \mathrm{~V}$ forward bias (filled circles) and 2 min later (open circles), respectively. The current density was approximately 20 $\mathrm{mA} / \mathrm{cm}^{2}$.

mer was found to enhance the transport and injection of holes from the anode into the emitting Alq layer. ${ }^{16}$

When an ITO/PTOPT/PBD/Al device is heated to about $100^{\circ} \mathrm{C}$ (by an external source or by excess heat produced by the current running through the device) an irreversible change of the electroluminescence spectrum occurs (Fig. 8). The peaks at 410 and $530 \mathrm{~nm}$ disappear and the peak at 610 $\mathrm{nm}$ is enhanced. Similar changes can also be observed in the photoluminescence spectrum of a glass/PTOPT/PBD structure when it is heated (Fig. 9). In the PL experiment the material was excited with a broad-band UV source with maximum intensity at $360 \mathrm{~nm}$. The PL of a PTOPT film excited with this UV-source is very weak. The peak at 610 $\mathrm{nm}$ appearing after heating can thus not be due to recombination of polaron excitons that are formed in the PTOPT. We therefore conclude that energy transfer from PBD to PTOPT takes place and that this transfer is enhanced after the heat treatment.

The PBD layer is transformed from an amorphous to a highly crystalline structure upon the heat treatment. This is verified by $x$-ray diffraction measurements of the samples. The temperature is in the same range as the melting temperature of PBD $\left(136-138^{\circ} \mathrm{C}\right)$ but well below the softening point of the polymer. The increased energy migration could thus be due to the crystallization of PBD, which increases the

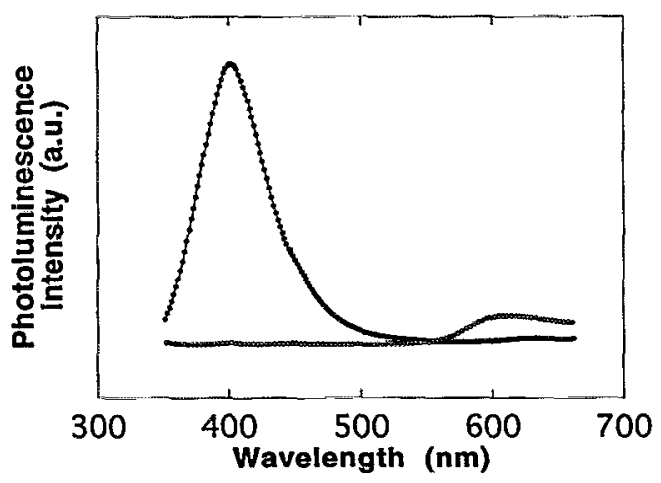

FIG. 9. Photoluminescence spectra of a glass PBD/PTOPT structure before (filled circles) and after (open circles) mild heat treatment in hot air.

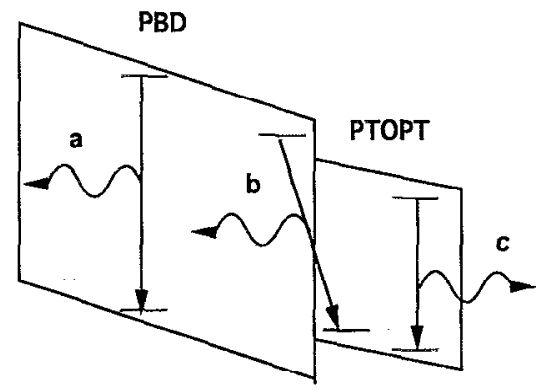

FIG. 10. Proposed mechanism for multiple emission: Exciton relaxation in PBD (a), exciton relaxation in PTOPT (c), and recombination of holes in PBD with electrons in PTOPT (b).

exciton mobility in the bulk of PBD, or to a reorganization at the PBD/PTOPT interface that enhances energy migration across the interface. After the heating process, there is no further change of the PL spectrum when the sample is cooled to room temperature.

It is not obvious that energy migration from $\mathrm{PBD}$ is the source of excitons in PTOPT also under EL conditions. PBD is a well known electron transport material and because of the large difference in band gap between the polymer and PBD ( $2 \mathrm{eV}$ vs $3.5 \mathrm{eV}$ ) there is probably no barrier for injection of electrons from PBD into PTOPT. It is therefore possible that electrons are present to a high degree in PTOPT and that polaron excitons are formed also in the polymer layer.

The change in the EL spectrum is correlated to an irreversible change in the $I-V$ and the EL- $V$ characteristics. More current is flowing through the device and more light is emitted at a certain voltage than before heating. No major change of thickness is seen during the thermal conversion of the PBD layer. We must conclude that the increased current is due to an increased mobility of electrons in PBD, or to a change of the interface between PBD and PTOPT which enhances the injection of electrons into PTOPT and/or holes into PBD.

To summarize this discussion we propose the following mechanism for light generation in this device (Fig. 10): Holes are injected from the ITO electrode into the heterostructure. The PTOPT layer acts as a transport layer and the holes drift to the PBD layer. A space charge layer is built up, which increases the electric field over the PBD layer. At a certain voltage the field over the PBD is high enough for electrons and holes to be injected into the PBD layer. In the PBD layer the electrons and holes combine to form singlet excitations which can decay radiatively in the PBD or migrate to the PTOPT layer. However, a certain fraction of electrons also reach the interface region. Here again there are two possibilities; either the electrons leave the PBD layer and form polaron excitons in the PTOPT or they recombine with hole-polarons at the interface and emit green light. Heating the device (by external source or by the heat evolved by the current passing through the device) induces a melting and crystallization of the PBD layer which significantly alters the conditions for energy and/or electron migration from 
PBD into the PTOPT. The emission from the device after the heat treatment is only that from polaron excitons in the PTOPT.

\section{CONCLUSIONS}

A thick molecule layer consisting of an oxadiazole derivative and a thin polythiophene derivative layer have been used to make a multiple emission peak heterostructure light emitting device. The emission spectrum consists of the colors blue $(410 \mathrm{~nm})$, green $(530 \mathrm{~nm})$, and red-orange $(620 \mathrm{~nm})$. The red and blue light is due to electroluminescence in the polymer layer and the molecule layer, respectively. The green peak probably originates from a transition between electron states in the molecule layer (PBD) and hole states in the polymer layer (PTOPT).

Different thicknesses of the oxadiazole layer tuned the threshold voltage for emission of light and double injection of charge carriers without changing the emission spectrum. Heat treatment of the device significantly changed the emission from the device from a two layer emitter to a one layer emitter. There is the possibility of tuning the relative intensity between the red, green, and blue peak by changing the interface between the PBD and PTOPT, or the morphology of the PBD.

Compared to a PTOPT device, the quantum efficiency was increased from $0.003 \%$ to $0.3 \%$ when a 1100 - $\AA$-thick oxadiazole layer was introduced.

\section{ACKNOWLEDGMENTS}

The $\mathrm{x}$-ray diffraction data were taken by Magnus Granström. This work was supported by the Swedish Engi- neering Research Council (TFR) and the Swedish National Board for Industrial and Technical Development (NUTEK).

${ }^{1}$ J. H. Burroughes, D. D. C. Bradley, A. R. Brown, R. N. Marks, K. Mackay, R. H. Friend, P. L. Burns, and A. B. Holmes, Nature 347, 539 (1990).

${ }^{2}$ D. Braun and A. J. Heeger, Appl. Phys. Lett. 58, 1982 (1991).

${ }^{3}$ G. Grem, G. Leditzky, B. Ullrich, and G. Leising, Adv. Matet. 4, 36 (1992),

${ }^{4}$ N. C. Greenham, S. C. Moratti, D. D. C. Bradley, R. H. Friend, and A. B. Holmes, Nature 365, 628 (1993).

${ }^{5}$ Y. Ohmori, M. Uchida, K. Muro, and K. Yoshino, Solid State Commun. 80, 605 (1991).

${ }^{6}$ C. W. Tang and S. A. Van Slyke, Appl. Phys. Lett. 51, 913 (1987).

${ }^{7}$ C. Adachi, S. Tokito, T. Tsutsui, and S. Saito, Jpn. J. Appl. Phys. 27, L713 (1988).

"S. Aratani, C. Zhang, K. Pakbaz, S. Hoger, F. Wudl, and A. J. Heeger, J. Electron. Mater. 22, 745 (1993).

${ }^{9}$ A. R. Brown, D. D. C. Bradley, J. H. Burroughes, R. H. Friend, N. C. Greenham, P. L. Burn, A. B. Holmes, and A. Kraft, Appl. Phys. Lett. 61, 2793 (1992).

${ }^{10} \mathrm{M}$. Uchida, Y. Ohmori, T. Noguchi, T. Ohnishi, and K. Yoshino, Jpn. J. Appl. Phys. 32, L921 (1993).

${ }^{11}$ J. Kido, K. Hogawa, K. Okuyama, and K. Nagai, Appl. Phys. Lett. 64, 815 (1994).

${ }^{12}$ M. R. Andersson, Q. Pei, T. Hjertberg, O. Inganäs, O. Wennerström, and J-E. Österholm (unpublished).

${ }^{13}$ E. Finkman, M. D. Sturge, and M. C. Tamargo, Appl. Phys. Lett. 49, 1299 (1986).

${ }^{14} G$. Abstreiter, in Two Dimensional Systems, Heterostructures and Superlattices, edited by G. Bauer, F. Kuchar, and H. Heinrich (Springer, Berlin, 1984).

${ }^{15} \mathrm{~J}$. A. Osaheni and S. A. Jenekhe, Macromolecules 27, 739 (1994),

${ }^{16} \mathrm{C}$. Hosokawa, H. Higashi, and T. Kusumoto, Appl. Phys. Lett. 62, 3238 (1993). 